W. Meier, C. Dem, C. Arndt, Mixing and reaction progress in a confined swirl flame undergoing thermoacoustic oscillations studied with laser Raman scattering, Experimental Thermal and Fluid Science 73 (2016) 71-78.

The original publication is available at www.elsevier.com

http://dx.doi.org/10.1016/j.expthermflusci.2015.09.011 


\title{
Mixing and reaction progress in a confined swirl flame undergoing thermo- acoustic oscillations studied with laser Raman scattering
}

\author{
W. Meier*, C. Dem, C.M. Arndt \\ Deutsches Zentrum für Luft- und Raumfahrt (DLR), Institut für Verbrennungstechnik, \\ Pfaffenwaldring 38-40, 70569 Stuttgart, Germany \\ *Corresponding author, wolfgang.meier@dlr.de \\ Keywords: gas turbine combustion, thermo-acoustic instabilities, laser Raman scattering
}

\begin{abstract}
A gas turbine model combustor for partially premixed flames was equipped with an optically accessible combustion chamber and operated with methane and air at atmospheric pressure, with a global equivalence ratio of 0.7 and a thermal power of $25 \mathrm{~kW}$. At these conditions the combustor exhibited thermo-acoustic oscillations with a frequency of approximately $400 \mathrm{~Hz}$. The flame behavior and its cyclic variations were investigated by laser Raman scattering for the simultaneous determination of the major species concentrations, temperature and mixture fraction. Additional information of the mean flow field and the flame shape was provided by particle image velocimetry and $\mathrm{OH}^{*}$ chemiluminescence imaging, respectively. Previously published results of phase-correlated mean values of this flame showed that the instability was sustained by the mechanism known as equivalence ratio fluctuations with convective delay. The current paper is focused on the characterization of the thermo-chemical state of the flame during the oscillation cycle. The mixture fraction varies considerably with spatial location and with the phase of the pressure oscillation and strong effects of turbulence-chemistry interaction are prevailing in the region close to burner mouth. Further, the effect of locally rich mixtures and elevated temperature on the local $\mathrm{CO}$ concentration level is shown.
\end{abstract}

\section{Introduction}

The performance of gas turbines (GT) for power generation and propulsion is often limited by the occurrence of thermo-acoustic instabilities which arise from the interaction of the flame dynamics with the acoustic modes of the combustion system [1,2]. Due to the complex coupling of flame stabilization, heat release, flow field and acoustic modes, a detailed understanding of the underlying mechanisms presents a difficult task, and a reliable prediction of frequencies and amplitudes of thermo-acoustic instabilities remains challenging. Periodic variations in heat release can stem from oscillations of the fuel stream entering the combustion chamber, equivalence ratio fluctuations or oscillations in flame area [1]. The varying thermal expansion associated with the heat release oscillations affects the pressure in the combustor which in turn influences the flow field. The relation between the time scales of the flow field variation and the acoustic modes of the system play a crucial role for the feedback mechanism of the thermo-acoustic instability. Another important aspect of the feedback mechanism concerns the response of the flame to variations of the flow field and chemical composition. The region where the flame can stabilize and the burning rate depend strongly on local strain rates and the composition and temperature of the reactants. Therefore, knowledge of the structure, flow field and thermo-chemical state of the flame as well as of their spatio-temporal fluctuations is essential for the understanding of the mechanisms leading to self-sustaining thermo-acoustic oscillations.

On the experimental side, good progress in this field has been achieved by the use of optically accessible GT model combustors and the application of optical and laser-based techniques, see e.g. Refs. [3,4] and the citations therein. Therefore, the Karlsruhe Institute of Technology (KIT) and the German Aerospace Center (DLR) have co-operatively designed 
and set up a GT model combustor with an optical combustion chamber for the investigation of thermo-acoustic combustion instabilities [5-7].

One of the target flames investigated was a partially premixed methane/air flame with an overall equivalence ratio of $\phi=0.7$ and a thermal power of $25 \mathrm{~kW}$. It exhibited thermoacoustic instabilities at a frequency around $400 \mathrm{~Hz}$ which coincided with an acoustic resonance of the plenum length. The flame and the phase-dependent variations during an oscillation cycle were previously studied by $\mathrm{OH}^{*}$ chemiluminescence imaging for the investigation of the cyclic variation of the heat release and overall flame characterization, stereoscopic particle image velocimetry (PIV) for the measurement of the flow field and by laser Raman scattering for the simultaneous determination of the major species concentrations, temperature and mixture fraction. The phase-correlated mean values from those measurements showed that the pressure variations in the combustion chamber modulated the flow field and equivalence ratio. Mixtures with alternating high and low fuel content were periodically formed in the burner nozzle near the fuel injection which then convected downstream towards the flame zone, where they caused a periodic variation of the heat release rate [7]. The reason for the varying fuel/air mixture in the nozzle was the different response (impedance) of the fuel and air supply lines to pressure variations. Arndt et al. [7] showed that the feedback loop of the self-sustaining combustion oscillation was closed by the coincidence of the convection time of the fuel-rich mixtures to the flame zone and the period of the acoustic resonance frequency. Thus, the instability was sustained by the mechanism known as equivalence ratio fluctuations with convective delay [8-12].

In the current paper, the focus is on mixing and reaction progress as well as on effects of turbulence-chemistry interaction in the same flame. The results are mainly based on singleshot laser Raman measurements which enabled the determination of the joint probability density functions of the concentrations of $\mathrm{CH}_{4}, \mathrm{O}_{2}, \mathrm{~N}_{2}, \mathrm{CO}_{2}, \mathrm{CO}, \mathrm{H}_{2} \mathrm{O}$ and $\mathrm{H}_{2}$, the mixture fraction and temperature. In addition, the mean flow field measured by particle image velocimetry, mean 2D distributions of $\mathrm{OH}^{*}$ chemiluminescence, mixture fraction and temperature are shown to yield a general overview of the flame.

\section{Experimental}

\subsection{Combustor}

Figure 1 shows a schematic of the combustor. The burner consists of an inner nozzle (diameter $\mathrm{D}=15 \mathrm{~mm}$ ) and a concentric annular nozzle (ID $=15.2 \mathrm{~mm}, \mathrm{OD}=24 \mathrm{~mm}$ ), which are supplied with dry air at room temperature from two separate plenums. Both flows are swirled in the same direction by individual radial swirl generators. The theoretical swirl number is $S_{o}=1.06$ for the outer swirler and $S_{i}=0.73$ for the inner swirler. Methane is supplied to the inner nozzle through 60 holes with a diameter of $0.5 \mathrm{~mm}$, arranged on a circumference at the wall $12 \mathrm{~mm}$ below the nozzle exit. Thus, the flow from the inner nozzle is partially premixed before entering the combustion chamber and reaching the flame zone. The flames are enclosed by a square combustion chamber (inner dimension $89 \mathrm{~mm} \times 89 \mathrm{~mm}$ cross section, $112 \mathrm{~mm}$ high) comprised of four fused silica plates which are held by steel frames. The frames are mounted to four posts in the corners and to the bottom and top of the combustion chamber. The resulting optical access to the flame is $73 \mathrm{~mm}$ wide and $112 \mathrm{~mm}$ high on each side. The origin of the coordinate system for all measurements is the center of the nozzle exit $(\mathrm{h}=0, \mathrm{r}=0)$. The exit of the upright combustion chamber is conically shaped leading to a short central exhaust pipe with an inner diameter of $50 \mathrm{~mm}$. Two of the four posts are equipped with ports for the installation of microphone probes. The lower part of the combustor contains the air plenums and supply lines. The housing is squared with inner dimensions of $90 \mathrm{~mm} \times 90 \mathrm{~mm}$. It has two tubes (ID =72 $\mathrm{mm}$ ) on the side for the air supplies. A defined acoustic boundary is achieved by sonic orifices located approximately $200 \mathrm{~mm}$ upstream of the inlet into the vertical plenum section. A round tube $(I D=50 \mathrm{~mm})$ along the 
axis of the confinement forms the inner boundary of the inner plenum. It contains the fuel tube (ID $=4 \mathrm{~mm}$ ) that terminates into a small plenum for the fuel distribution at the nozzle. The inner air plenum has a height of $380 \mathrm{~mm}$, a round cross section with an ID of $50 \mathrm{~mm}$ and an OD of $76 \mathrm{~mm}$ at its upper half and a square $90 \mathrm{~mm} \times 90 \mathrm{~mm}$ cross section at its lower half. The outer air plenum has a height of $200 \mathrm{~mm}$, an ID of $80 \mathrm{~mm}$ and an outer boundary of 90 $\mathrm{mm} \times 90 \mathrm{~mm}$. Ports for microphone probes enable the registration of acoustic oscillations in the plenums as indicated Fig. 1.

The flow rates were metered by electromechanical mass flow meters (Brooks type 5851S for $\mathrm{CH}_{4}$ and type SLA5853S for each air flow) and additionally controlled by calibrationstandard Coriolis mass flow meters (Siemens SITRANS F C MASS 2100 DI 3 for $\mathrm{CH}_{4}$, Siemens SITRANS F C MASS 2100 DI 15 for each air flow) with an accuracy of approx. 1.5 $\%$.

The burner was operated with 451 and $282 \mathrm{~g} / \mathrm{min}$ of air in the outer and inner plenum, respectively, and $30 \mathrm{~g} / \mathrm{min}$ of $\mathrm{CH}_{4}$. The corresponding thermal power was $25 \mathrm{~kW}$ and the global equivalence ratio $\phi=0.7$. More details of the combustor are given in Ref. [7]. The pressure oscillations in the combustion chamber and in the two air plenums were measured with calibrated microphone probes (Brüel\&Kjær, type 4939) with a sampling rate of $50 \mathrm{kHz}$.

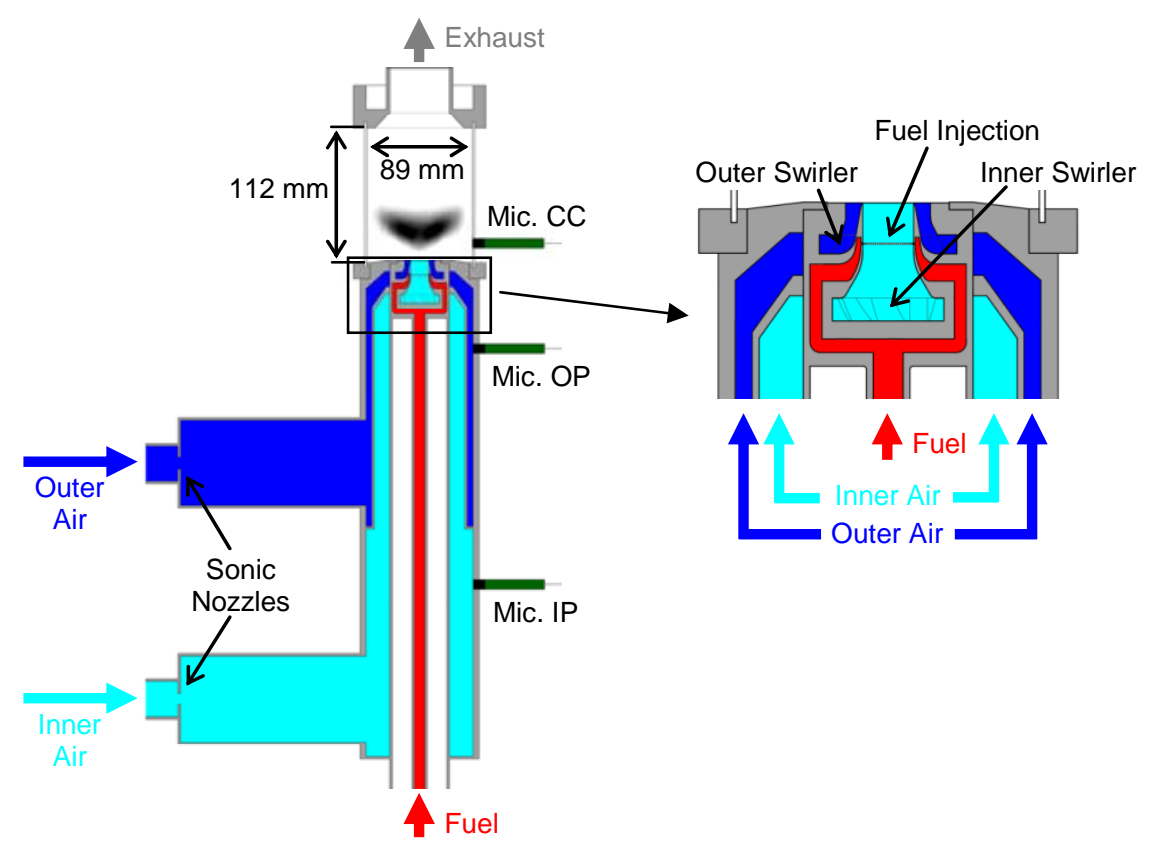

Figure 1. Schematic of the combustor.

\subsection{Laser Raman scattering}

Single-shot laser Raman scattering was applied for the pointwise quantitative measurement of the major species concentrations $\left(\mathrm{O}_{2}, \mathrm{~N}_{2}, \mathrm{CH}_{4}, \mathrm{H}_{2}, \mathrm{CO}, \mathrm{CO}_{2}, \mathrm{H}_{2} \mathrm{O}\right)$ and the temperature. The measurement system has been described previously $[11,13,14]$ and only a brief summary is given here. The radiation of a flashlamp-pumped dye laser (Candela LFDL 20, wavelength $\lambda$ $=489 \mathrm{~nm}$, pulse energy $\mathrm{E}_{\mathrm{p}} \approx 1.8 \mathrm{~J}$, pulse duration $\tau_{\mathrm{p}} \approx 3 \mu \mathrm{s}$, pulse repetition rate $5 \mathrm{~Hz}$ ) was focused into the combustion chamber and the Raman scattering emitted from the measurement volume (length $\approx 0.6 \mathrm{~mm}$, diameter $\approx 0.6 \mathrm{~mm}$ ) was collected by an achromatic lens $(\mathrm{D}=80 \mathrm{~mm}, \mathrm{f}=160 \mathrm{~mm})$ and relayed to the entrance slit of a spectrograph (SPEX 1802, $\mathrm{f}=1 \mathrm{~m}$, slit width $2 \mathrm{~mm}$, dispersion $\approx 0.5 \mathrm{~nm} / \mathrm{mm}$ ). The dispersed and spatially separated signals from the different species were detected by individual photomultiplier tubes and sampled using boxcar integrators. The species number densities were calculated from the 
signals using calibration measurements and the temperature was deduced from the total number density via the ideal gas law. The simultaneous detection of all major species with each laser pulse also enabled the determination of the instantaneous mixture fraction [15]. The mixture fraction is the ratio of the mass in a sample originating from the fuel stream (here: all $\mathrm{C}$ and $\mathrm{H}$ atoms) to the total mass.

Measurements were performed at different heights above the burner nozzle and at various radial locations. Measurement locations with $\mathrm{h}<8 \mathrm{~mm}$ and $\mathrm{r}>27 \mathrm{~mm}$ were not accessible due to clipping of the solid angle of the detection optics. At locations with substantial variation of the temperature and species concentrations during a cycle of the thermo-acoustic oscillation the measurements were performed with phase-correlation (described below). In that case, approximately 2500 single-shot measurements were recorded at each radial location at $\mathrm{h}=8,25$ and $40 \mathrm{~mm}$ and approximately 1750 single shots at each radial location at $\mathrm{h}=15$ $\mathrm{mm}$. From these the phase-correlated joint probability density functions (PDFs) of the major species mole fractions, mixture fraction, and temperature were constructed. At $h>40 \mathrm{~mm}$ phase-dependent changes were so small that measurements were performed without phasecorrelation.

The dominant thermo-acoustic frequency of the flame investigated was around $400 \mathrm{~Hz}$. To correlate the values of the laser measurements with the phase-angle of the pressure oscillation cycle, acoustic and single-shot results were recorded simultaneously so that each single-shot measurement could be assigned a pressure value from the combustion chamber. In

a post-processing step, the measured values were assorted with respect to the phase-angle and grouped within eight bins, each comprising $45^{\circ}$ phase angle span. Phase angle $0^{\circ}$ was defined as the transition from negative to positive pressure fluctuation in the combustion chamber. Accordingly, the range between $-22.5^{\circ}$ and $+22.5^{\circ}$ was defined as "phase 1", the range between $22.5^{\circ}$ and $67.5^{\circ}$ "phase 2 " etc.

With respect to measurement uncertainties, it must be distinguished between systematic errors arising from, for example, uncertainties in the calibration procedure, and statistical errors which are mainly caused by the statistics (shot noise) of the detected Raman photons in a single-shot measurement. Systematic uncertainties were typically $\pm 3-4 \%$ for the temperature and mixture fraction, $\pm 3-5 \%$ for the mole fractions of $\mathrm{O}_{2}, \mathrm{H}_{2} \mathrm{O}, \mathrm{CO}_{2}$ and $\mathrm{CH}_{4}$. Because of the low concentrations of $\mathrm{H}_{2}$ and $\mathrm{CO}$ in the flame investigated, the uncertainty is relatively large for these species. It is estimated to be around $\pm 20 \%$ at a mole fraction of 0.01 . Typical statistical uncertainties (for a single-shot measurement) were $3-3.5 \%$ for the temperature and mixture fraction, $4 \%$ for $\mathrm{H}_{2} \mathrm{O}$ and $9 \%$ for $\mathrm{O}_{2}$ and $\mathrm{CO}_{2}$ in the exhaust gas.

\subsection{Particle image velocimetry (PIV)}

The details of the particle image velocimetry (PIV) measurement system are given in Ref. [7] and only a brief description is presented here. All three velocity components were measured using stereoscopic PIV. The system (FlowMaster, LaVision) consisted of a frequencydoubled dual-head Nd:YAG laser (NewWave Solo 120), two CCD cameras (LaVision Imager Intense, $1376 \times 1040$ pixels), operated in double-frame mode, and a programmable timing unit (PTU 9, LaVision). The laser sheet covered the central vertical plane of the combustion chamber and had a thickness of around $1 \mathrm{~mm}$. The cameras were equipped with a wide-angle lens $(\mathrm{f}=16 \mathrm{~mm})$, a bandpass filter $(532 \pm 5 \mathrm{~nm})$ and were mounted on Scheimpflug adapters to align their focal plane with the laser sheet. Both fields of view covered an area $-39 \mathrm{~mm}<\mathrm{r}<$ $39 \mathrm{~mm}$ and $0.5 \mathrm{~mm}<\mathrm{h}<105 \mathrm{~mm}$. The air flow was seeded with titanium dioxide particles with a nominal diameter of $1 \mu \mathrm{m}$. The velocity fields were evaluated using a commercial PIV software (LaVision Davis 8.0). A multi-scale cross-correlation algorithm was used with a final interrogation window size of $16 \times 16$ pixel, corresponding to an in-plane spatial resolution of $1.5 \times 1.5 \mathrm{~mm}^{2}$, and a window overlap of $50 \%$. 


\subsection{Chemiluminescence imaging}

$\mathrm{OH}^{*}$ chemiluminescence was recorded with an intensified high speed CMOS camera (LaVision HSS 5 with LaVision HS-IRO, active array 512 × 512 pixel), equipped with a fast UV lens ( $\mathrm{f}=45 \mathrm{~mm}, \mathrm{f} / 1.8$, Cerco) and a high-transmission bandpass filter (Transmission $>$ $80 \%$ at $310 \mathrm{~nm}$ ). The field of view was $-39 \mathrm{~mm}<\mathrm{r}<39 \mathrm{~mm}$ and $0 \mathrm{~mm}<\mathrm{h}<75 \mathrm{~mm}$. The mean distribution presented in this paper was averaged over 8200 individual images recorded at a frame rate of $10 \mathrm{kHz}$ with an exposure time of $15 \mu \mathrm{s}$. The images were dark frame and flat-field corrected. This technique yields line-of-sight integrated signals, however, spatially resolved information can be gained by deconvolution, taking advantage of the statistical rotational symmetry of the flame. This results in a "quasi 2-D image" of the center plane, where the local distribution of the chemiluminescence can be identified more clearly than in the integral view. More details of the measurements are described in Ref. [7].

\section{Results}

\subsection{Flow field and flame zone}

To yield a general impression of the flow field, Fig.2 shows a streamline plot of the velocity field averaged over all phase angles. The colors represent the magnitude of the axial velocity component; the white lines are the boundaries of the recirculation zones (zero axial velocity) and the rectangle framed by the dotted line is the region where Raman measurements have been performed. The flow field is typical of a swirl flame with a conically shaped region of the inflow, an inner and outer recirculation zone, IRZ and ORZ, respectively, and the corresponding shear layers between the inflow and the recirculation zones. Although the inflow is supplied from two nozzles, the flows from the two nozzles are hardly distinguishable because they merge very quickly [7].

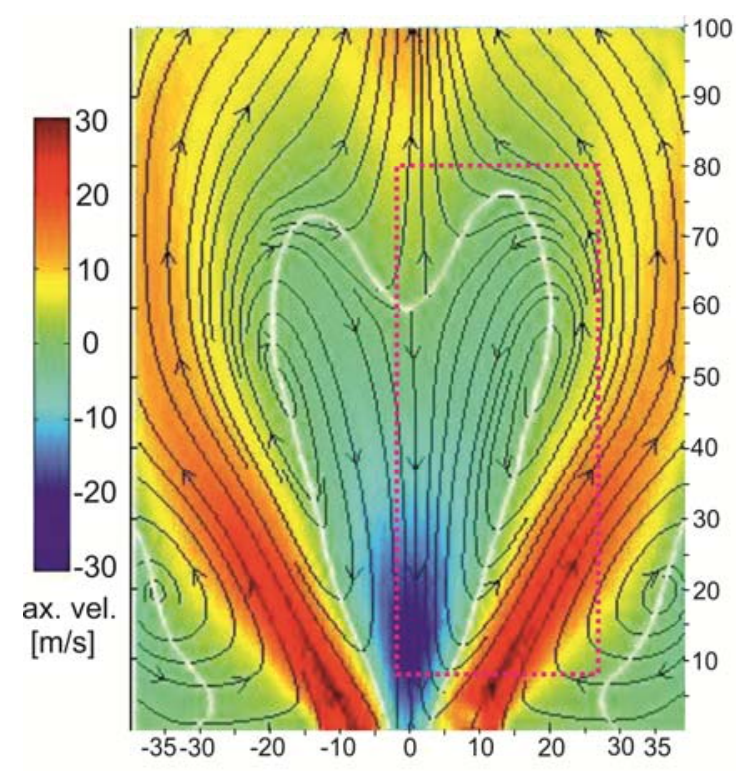

Figure 2. Streamline plot of the mean flow field. The axial velocity component is colorcoded. The white lines correspond to zero mean axial velocity and the dotted line indicates the region of Raman measurements. Scales are in $\mathrm{mm}$.

Instantaneous velocity distributions are, of course, significantly different from the mean distribution and are dominated by turbulent structures, particularly by unsteady vortices in the inner shear layer [7]. Phase-dependent changes of the flow field were revealed by phasecorrelated mean values, i.e. by single-shot distributions averaged within distinct phase intervals. They showed that during a cycle of the thermo-acoustic oscillation, the magnitude 
of all three velocity components varied considerably and that also the size and shape of the recirculation zones changed [7].

The flame shape has been visualized by $\mathrm{OH}^{*}$ chemiluminescence imaging. An averaged chemiluminescence distribution and the corresponding inverse Abel-transformed distribution are displayed in Fig.3. The flame has a conical shape and the region of flame anchoring starts approximately $8 \mathrm{~mm}$ above the nozzle. The flame extends to a height of $\mathrm{h} \approx 50 \mathrm{~mm}$. The high intensities seen at the combustor walls in the Abel-transformed distribution mostly stem from the red-hot posts of the combustion chamber and from reflections at the windows and are exaggerated by the Abel transformation. The phase-correlated mean values which are presented in Ref. [7] showed that the intensity of the $\mathrm{OH}^{*}$ distribution varied significantly during an oscillation cycle while the shape of the distribution was almost unaffected. In particular, the region where the flame anchored was rather fixed on the flame axis at $h \approx 8$ $\mathrm{mm}$. This indicates that the thermo-chemical state of the flame must have been quite favorable for flame stabilization throughout the thermo-acoustic oscillation. Therefore, the thermochemical state, as characterized by the single-shot Raman measurements, will be examined in the following.

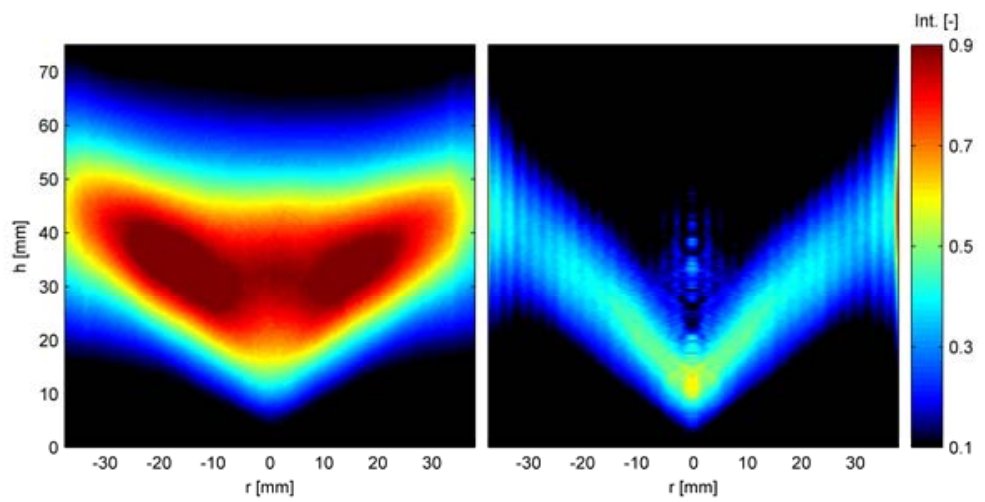

Figure 3. Averaged normalized $\mathrm{OH}^{*}$ chemiluminescence distribution, line-of-sight integrated (left) and Abel-transformed (right).

\subsection{Mean mixture fraction and temperature}

Figure 4 shows the 2D distributions of the mixture fraction, $f_{\text {avg, }}$, and the temperature, $\mathrm{T}_{\mathrm{avg}}$. They have been constructed by interpolation between the locations where Raman measurements have been performed and they have been averaged over all single shots at all phases. The distribution of the mixture fraction $\mathrm{f}_{\text {avg }}$ shows fuel-rich mixtures above the central nozzle $(\mathrm{r}<12 \mathrm{~mm}$ at $\mathrm{h}=8 \mathrm{~mm})$ and very lean fluid above the annular nozzle $(\mathrm{r}>12 \mathrm{~mm}$ at $\mathrm{h}$ $=8 \mathrm{~mm})$. This reflects the operation of the nozzles with fuel/air mixtures and pure air, respectively. Accordingly, the mixtures in the ORZ are leaner than those in the IRZ. The gradients are quickly reduced by turbulent mixing. The corresponding distribution of the temperature $\mathrm{T}_{\text {avg }}$ reflects the mean reaction progress and demonstrates that the IRZ conveys hot gas to the flame anchoring region. The highest mean temperatures are nearly $1890 \mathrm{~K}$. In the ORZ, the mean temperatures are significantly lower than in the IRZ. It should be kept in mind that the region covered by the Raman measurements reaches only up to $r=27 \mathrm{~mm}$ while the combustion chamber extends to $r=44.5 \mathrm{~mm}$.

\subsection{Correlated results of mixture fraction and temperature}

Figure 5 displays a scatterplot of temperature vs. mixture fraction at the lowest height of the Raman measurements $(\mathrm{h}=8 \mathrm{~mm})$. Each dot represents the result of a single-shot measurement, and results from different radial locations are marked by different colors. For comparison, the solid line represents the thermo-chemical state of a strained laminar 

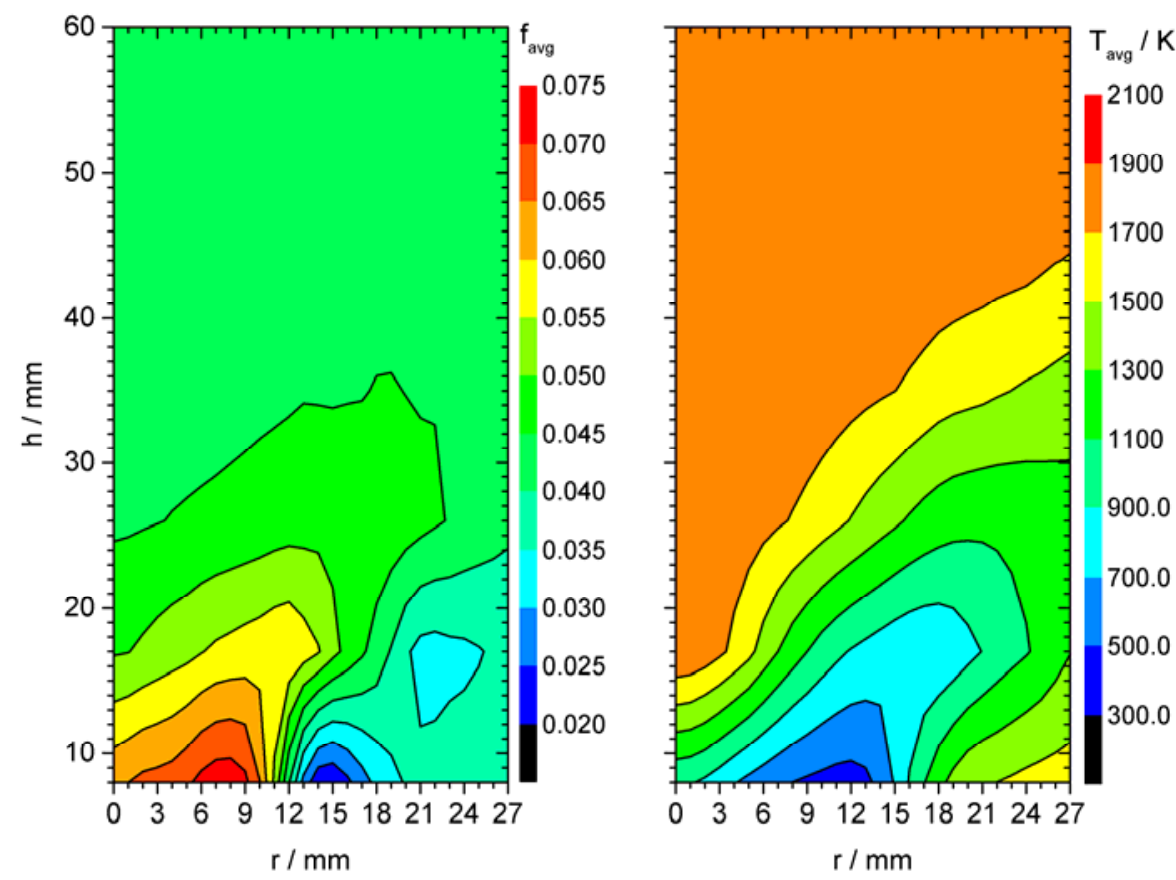

Figure 4. Averaged distributions of mixture fraction $\mathrm{f}_{\text {avg }}$ (left) and temperature $\mathrm{T}_{\text {avg }}$ (right). For comparison, the stoichiometric mixture fraction is 0.055 and global mixture is 0.0391 .

counterflow diffusion flame in Tsuji geometry for a strain rate of $a=1 \mathrm{~s}^{-1}$ [16]. At this strain rate, the state of the flame is close to adiabatic equilibrium. Although the partially premixed turbulent flame investigated cannot be directly compared to a counterflow diffusion flame, the calculated curve may serve here as an aid for assessing the thermo-chemical state of the flame under investigation. The vertical dashed lines indicate the stoichiometric mixture fraction $\mathrm{f}_{\text {stoich. }}=0.055$ and the global mixture fraction $\mathrm{f}_{\text {global }}=0.0391$ deduced from the total flow rates.

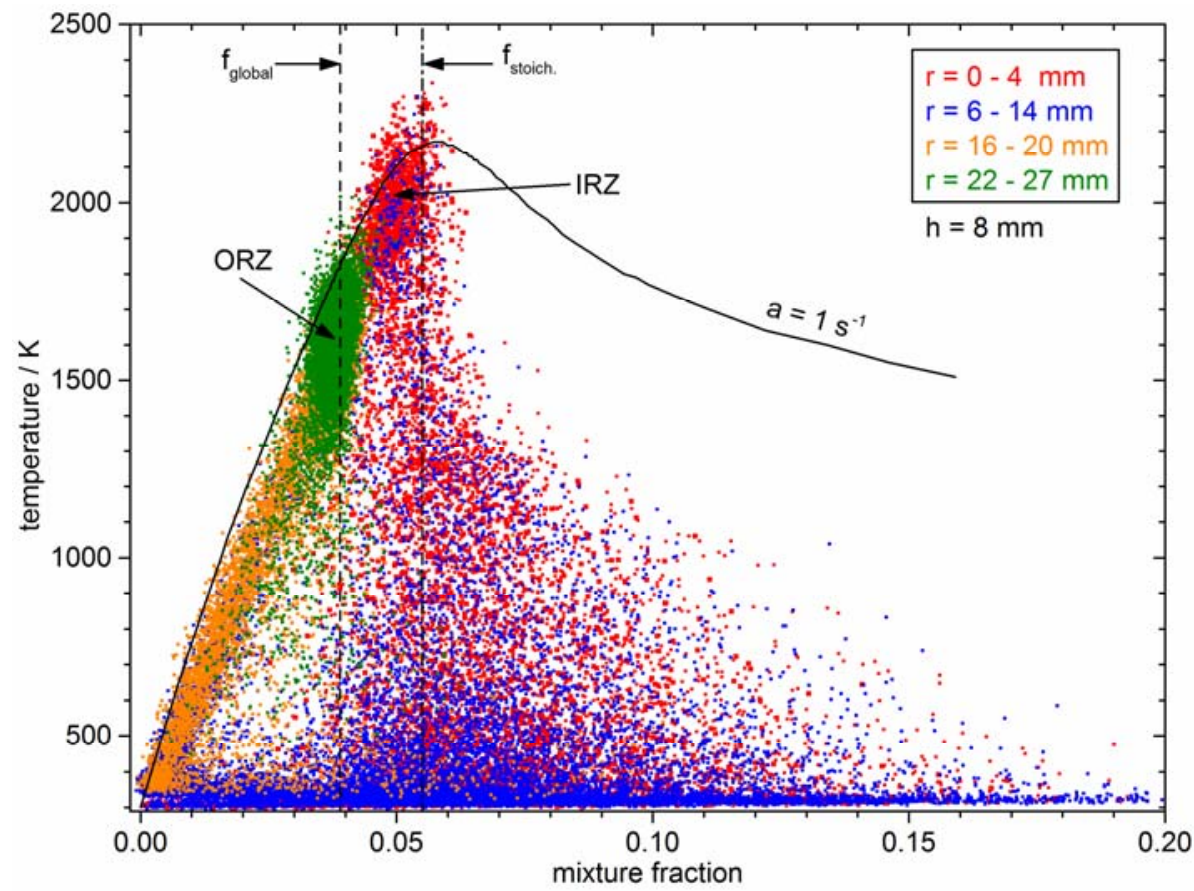

Figure 5. Correlation (scatterplot) between temperature and mixture fraction at $\mathrm{h}=8 \mathrm{~mm}$. 
The samples from the radial locations with $\mathrm{r} \geq 22 \mathrm{~mm}$ are predominantly from the ORZ. They are close to the global mixture fraction and the observed scatter in mixture fraction is largely caused by the measurement precision. The measured temperatures are slightly below the calculated values for $\mathrm{a}=1 \mathrm{~s}^{-1}$ (and adiabatic flame temperature). An inspection of the species composition shows that the flame is at chemical equilibrium in this region and that the temperature decrease is most likely due to heat loss of the combustion gases at the burner plate and the combustor walls during the transport in the ORZ. The samples in the adjacent radial region ( $\mathrm{r}=16-20 \mathrm{~mm}$, outer shear layer) present mixtures of the gas from the ORZ and the air from the annular nozzle. These samples contain no fuel and the state is solely determined by mixing. The radial region between $\mathrm{r}=6$ and $14 \mathrm{~mm}$ is dominated by the inflow. Here, the scatter in mixture fraction extends from pure air $(\mathrm{f}=0)$ to rich mixtures (up to $\mathrm{f} \approx 0.2$ or $\phi \approx 4.3$ ). Many of these samples are at or close to room temperature and contain only small amounts of combustion products, i.e. they represent fresh gas from the nozzles with possible admixtures of exhaust gas from the recirculation zones. The most interesting region is close to the flame axis $(\mathrm{r}=0-4 \mathrm{~mm})$ where the IRZ and the shear layer between the inflow and the IRZ reside and where the flame is anchored. Here, a large number of samples exhibits high temperatures and mixture fractions between $f_{\text {global }}$ and $f_{\text {stoich. }}$. They are close to the calculated thermo-chemical state and are either recirculated hot gas or combustion products which have just been formed in the nearby flame zone. The remainder of the samples from this area exhibits a large variation in temperature and reaction progress. The corresponding distributions of species concentrations show that these samples contain $\mathrm{CH}_{4}$, air and exhaust gas often together with $\mathrm{CO}$ and $\mathrm{H}_{2}$. These samples are most likely mixtures of exhaust gas from the IRZ and fresh fuel/air mixtures from the central nozzle which have not reacted yet due to ignition delay. Such behavior has often been observed in flames with recirculating burned gas [11,17-23] and an estimation of the ignition delay times with the time scale of the flow field makes this interpretation plausible [18]. Additionally, events of local flame extinction can lead to similar thermo-chemical states but are not expected to such large extent in this region [24].

In the following, the phase-dependent variations of the T-f-correlations are considered. In Fig. 6 scatterplots from $\mathrm{h}=8 \mathrm{~mm}$ are displayed at four different phases of the thermo-acoustic cycle. Only samples from the radial region $r=0-10 \mathrm{~mm}$ are included in order to focus on the shear layer between the IRZ and the inflow. The definition of the phases is the same as in Ref. [7] so that the results presented here can be combined with those. However, for the sake of brevity only four phases are shown here. In addition to the single-shot values, ensembleaveraged values are displayed by crosses for the samples from the radial region $r=0-4 \mathrm{~mm}$ (black/red crosses) and $\mathrm{r}=6-10 \mathrm{~mm}$ (black/blue crosses). Phase 4 is characterized by a large number of very lean and cold samples and a rather small number of samples with high temperatures. The IRZ is well developed at this phase and extends to a radial position of up to $\mathrm{r} \approx 7 \mathrm{~mm}$ (not displayed); however, the scatterplots show that it contains only small amounts of hot burned gas. The portion of lean samples is relatively large, but the average mixture faction is slightly rich. The small number of samples representing burned gas demonstrates that the reaction progress is small. The opposite is seen at phase 8 , where the distribution is shifted to rich mixtures and the reaction progress is greater. The distributions at the phases 2 and 6 lie between the extremal values. The scatterplots reflect quantitatively the phasedependent mixture fraction fluctuations which are associated with the pressure variations in the combustion chamber and which are caused by the different response of the fuel and air supply lines of the combustor as discussed in the introduction. While the evaluation of the phase-dependent mean values of the species distribution and flow velocities described in Ref. [7] lead to the same conclusion, the scatterplots additionally illustrate the reaction progress and details of the PDFs. 

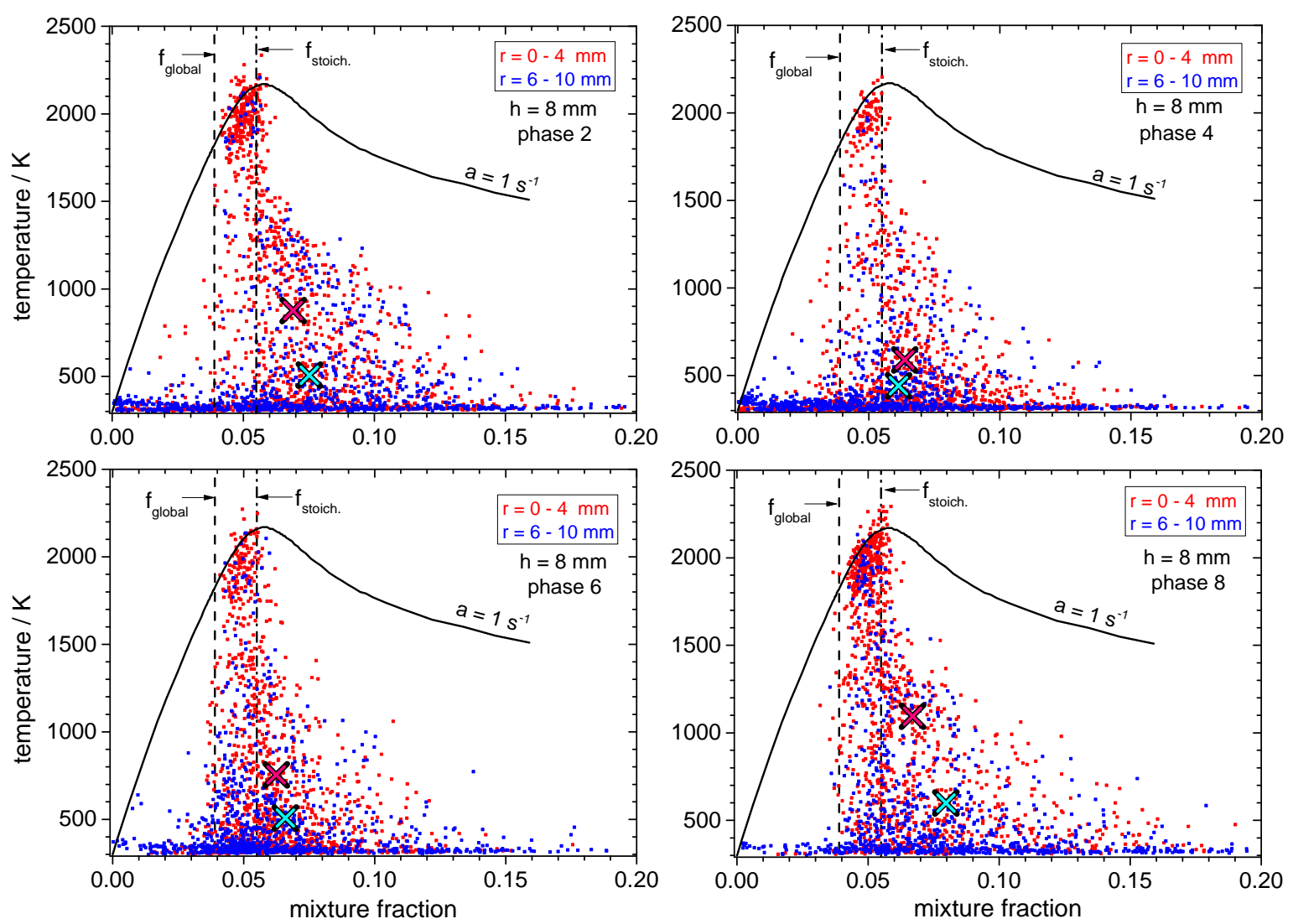

Figure 6. Correlation (scatterplot) between temperature and mixture fraction at $\mathrm{h}=8 \mathrm{~mm}$. The crosses indicate the average values: The black/red ones for samples from $r=0$ to 4 $\mathrm{mm}$, the black/blue ones for samples from $\mathrm{r}=6$ to $10 \mathrm{~mm}$.

Because the flame anchoring region is located around $\mathrm{h}=8 \mathrm{~mm}$, there is little probability of finding a reaction zone at this height and the thermo-chemical state is mainly determined by mixing. However, in order to initiate flame stabilization in the vicinity of this location, the thermo-chemical state and the flow field must fulfill certain requirements. In particular, nearstoichiometric mixtures and elevated temperatures should be present at a sufficient frequency to enable high flame propagation speeds. Although the scatterplots in Fig.6 exhibit largely different mean temperatures at the phases considered, there are frequently samples present at all phases which provide optimal conditions for flame stabilization. This explains why the flame anchoring region does not move significantly during an oscillation cycle.

At $\mathrm{h}=15 \mathrm{~mm}$ the flame zone extends from $\mathrm{r}=0$ to about $\mathrm{r}=10-15 \mathrm{~mm}$ (see Fig.3). Scatterplots of temperature vs. mixture fraction for this region $(\mathrm{h}=15 \mathrm{~mm}, \mathrm{r}=0-15 \mathrm{~mm})$ are displayed in Fig.7 for the same four phases as in Fig.6. It is seen that mixing and reaction have significantly progressed, but there is still a large number of samples with intermediate states between non-reacted and fully reacted. Also, most of the samples from this region are fuel-richer than the global mixture fraction and a large number exhibits mixture fractions larger than $f_{\text {stoich. }}$. The ensemble-averaged values (marked by the crosses) are very close to the stoichiometric mixture fraction. As at $\mathrm{h}=8 \mathrm{~mm}$, there are many samples with increased temperatures at all phases with best conditions for flame stabilization.

It can thus be concluded that flame reactions take predominantly place at nearstoichiometric conditions, or, in other words, that the flame finds optimal compositional conditions at this height. Compared to the scatterplots at $\mathrm{h}=8 \mathrm{~mm}$, the order of the phases with respect to large mixture fraction values has changed. For example, at $h=8 \mathrm{~mm}$, the samples from phase 8 tended to be fuel-richer while at $\mathrm{h}=15 \mathrm{~mm}$, those from phase 4 exhibit more fuel-rich mixtures. This is explained by the convective transport of fuel-rich fluid during an oscillation cycle and reflects the convective delay that is part of the feedback mechanism 
of the thermo-acoustic instability. A more detailed analysis of the convective delay that includes eight phases and all radial positions as well as the flow velocity has been presented in Ref. [7].
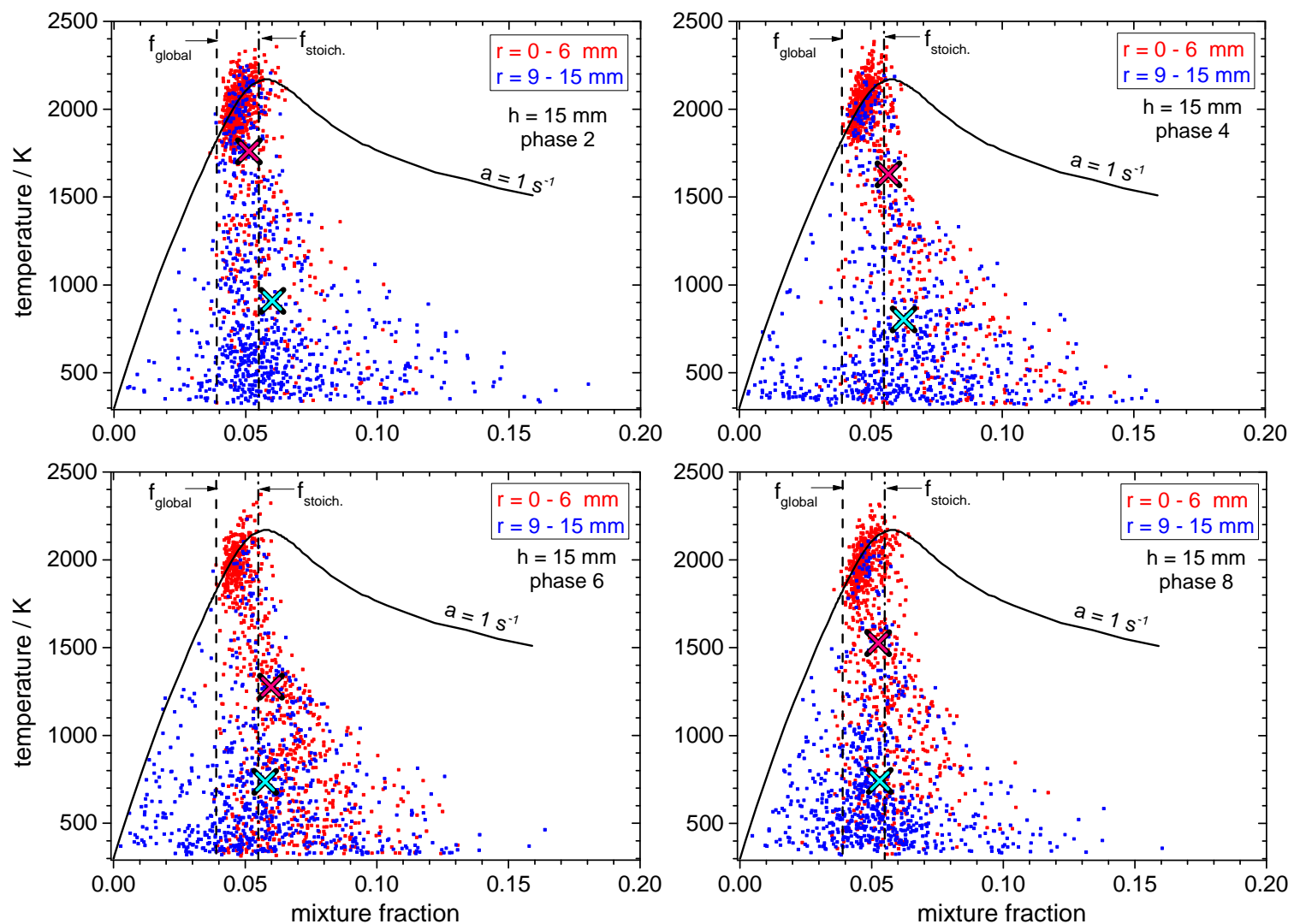

Figure 7. Correlation (scatterplot) between temperature and mixture fraction at $\mathrm{h}=15$ $\mathrm{mm}$. The black/red crosses indicate the average values for samples from $\mathrm{r}=0$ to $6 \mathrm{~mm}$, the black/blue ones for samples from $r=9$ to $15 \mathrm{~mm}$.

Due to the frequent occurrence of fuel-rich mixtures local $\mathrm{CO}$ concentrations can be relatively high. Figure 8 presents phase-correlated ensemble-averaged values of the $\mathrm{CO}$ mole fractions at the four phases shown in Figs. 6 and 7 at $\mathrm{h}=8$ and $15 \mathrm{~mm}$. At $\mathrm{h}=8 \mathrm{~mm}$ well pronounced phase-dependent variations of the $\mathrm{CO}$ concentration levels are observed that correlate with the scatterplots of Fig. 6 in the sense that at phases with a large number of fuelrich samples (phases 8 and 2) also large $\mathrm{CO}$ concentrations are observed. Also, the largest phase-dependent variations and total concentrations are observed at $0 \leq \mathrm{r} \leq 4 \mathrm{~mm}$, corresponding to the region where intermediate reaction states occur most frequently, as shown in Fig.6. At $\mathrm{h}=15 \mathrm{~mm}$ the mean $\mathrm{CO}$ mole fraction has considerably grown to maximum values of approximately 0.014 . A phase-dependence is not clearly evident. At $\mathrm{h}=$ $25 \mathrm{~mm}$ (not displayed) the $\mathrm{CO}$ mole fractions are reduced to maximum mean values on the order of 0.001 . Thus, $\mathrm{CO}$ is rapidly formed and consumed depending on the local thermochemical state. 

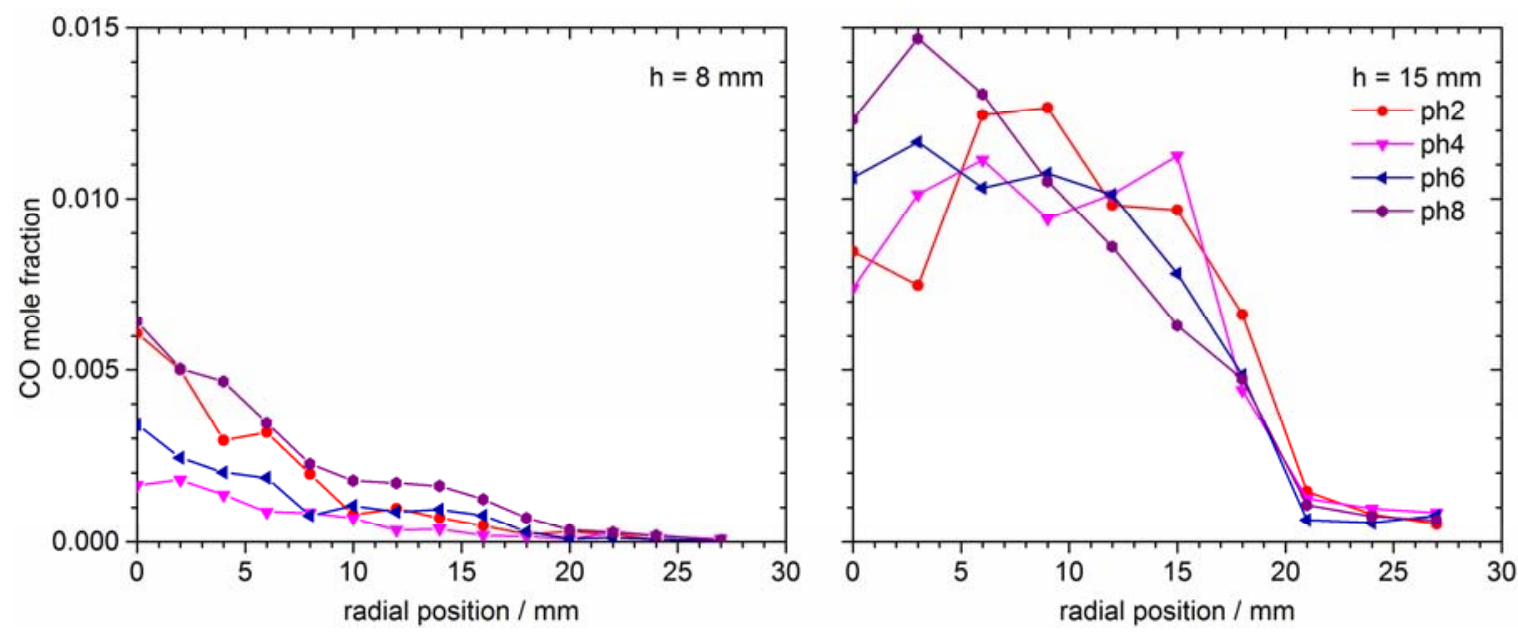

Figure 8. Radial profiles of phase-correlated mean values of the $\mathrm{CO}$ mole fraction for 4 phases at $\mathrm{h}=8$ and $15 \mathrm{~mm}$.

\section{Summary and Conclusion}

A partially premixed methane/air swirl flame has been operated under conditions at which thermo-acoustic instabilities at a frequency of approximately $400 \mathrm{~Hz}$ occurred. The frequency corresponded to an acoustic resonance of the inner plenum chamber in such a way that half of the acoustic wavelength (based on the speed of sound in cold air) coincided with the length of the plenum. In a previous publication it has been demonstrated that the mechanism of the instability is based on equivalence ratio fluctuations in the central nozzle which reach the flame zone after a convective delay that corresponds to the period of the oscillation frequency. The current study was focused on the thermo-chemical state of the flame and the mixing and reaction progress.

The single-shot laser Raman measurements revealed a wide range of mixing and reaction progress in the region near the nozzle. Within the recirculation zones, burned gas close to chemical equilibrium was prevailing while the inflow contained mostly non-reacted mixtures near room temperature. In the shear layer between the inflow and the inner recirculation zone, the samples covered the complete range of thermo-chemical states between non-reacted and completely reacted. The samples with intermediate temperatures contained fuel, air and exhaust gas. They were explained as mixtures of inflowing fresh gas and recirculating hot gas which had not yet reacted due to ignition delay.

The temperature and mixture fraction distributions varied during an oscillation cycle in response to the pressure variations in the combustion chamber. The observed cyclic variations in mixture fraction reflected the periodic formation of larger and smaller fuel clouds in the central nozzle. The phase shift of the mixture fraction variation between $\mathrm{h}=8$ and $15 \mathrm{~mm}$ was a result of the convective delay of the fuel-rich fluid.

From the mean chemiluminescence distribution it was seen that the flame was anchored at a height of $\mathrm{h} \approx 8 \mathrm{~mm}$ near the flame axis. The scatterplots from this region showed a large number of samples with near-stoichiometric mixture fractions and elevated temperatures that present very good conditions for flame stabilization. This explains the flame anchoring in this region. Together with the chemiluminescence distributions, the scatterplots revealed that at $h$ $=15 \mathrm{~mm}$ flame reactions took predominantly place at near-stoichiometric conditions. The presence of fuel-rich mixtures and elevated temperatures at $\mathrm{h}=15 \mathrm{~mm}$ caused a (transient) occurrence of abundant $\mathrm{CO}$. 


\section{Acknowledgement}

The financial support by the German Research Council (DFG) within the Collaborative Research Center 606 is gratefully acknowledged.

\section{References}

[1] Lieuwen T.C., Yang V., Combustion Instabilities in Gas Turbine Engines: Operational Experience, Fundamental Mechanisms, and Modeling, American Institute of Aeronautics and Astronautics, Inc., Reston, Virginia, 2006.

[2] Candel S., Durox D., Schuller T., Bourgouin J.-F., Moeck J.P., "Dynamics of Swirling Flames“, Ann. Rev. Fluid Dynamics 46:147-173 (2014).

[3] Dhanuka S.K., Temme J.E., Driscoll J.F., "Unsteady Aspects of Lean Premixed Prevaporized Gas Turbine Combustors: Flame-Flame Interactions", J. Propulsion Power 27: 631-641 (2011).

[4] Stöhr M., Boxx I., Carter C.D., Meier W., "Experimental study of vortex-flame interaction in a swirl combustor", Combust. Flame 159: 2636-2649 (2012).

[5] Kraus C., Bockhorn H., "Experimental and numerical investigation on combustion instabilities in swirl-stabilized flames operated in partially-premixed mode", Proc. European Combustion Meeting, Lund, Sweden, paper P5-26 (2013).

[6] Bärow E., Koch R., Bauer H.-J., "Comparison of oscillation modes of spray and gaseous flames", Proc. Eighth Mediterranean Combustion Symposium, Cesme, Izmir, Turkey, paper EGTSC-26 (2013).

[7] Arndt C.M., Severin M., Dem C, Stöhr M., Steinberg A.M., Meier W., "Experimental analysis of thermo-acoustic instabilities in a generic gas turbine combustor by phasecorrelated PIV and laser Raman scattering”, Exp. Fluids 56, 69 (2015).

[8] Lieuwen T., Torres H., Johnson C., Zinn B.T., "A Mechanism of Combustion Instability in Lean Premixed Gas Turbine Combustors", Transaction of the ASME 123: 182-189 (2001).

[9] Sattelmayer T., "Influence of the Combustor Aerodynamics on Combustion Instabilities from Equivalence Ratio Fluctuations", J. Eng. Gas Turbines Power 125:11-19 (2003).

[10] Schürmans B., Bellucci V., Güthe F., Meili F., Flohr P., Paschereit C.O., "A detailed Analysis of thermoacoustic interaction mechanism of a turbulent premixed flame", Proc. ASME Turbo Expo, Vienna, Austria, paper GT2004-53831 (2004).

[11] Meier W., Weigand P., Duan X.R., Giezendanner-Thoben R., "Detailed Characterization of the Dynamics of Thermoacoustic Pulsations in a Lean Premixed Swirl Flame", Combust. Flame 150:2-26 (2007).

[12] Cosic B., Terhaar S., Moeck J.P., Paschereit C.O., "Response of a swirl-stabilized flame to simultaneous perturbations in equivalence ratio and velocity at high oscillation amplitudes", Combust. Flame 162:1046-1062 (2015).

[13] Keck O., Meier W., Stricker W., Aigner M., "Establishment of a Confined Swirling Natural Gas/Air Flame as a Standard Flame: Temperature and Species Distributions from Laser Raman Measurements", Combust. Sci. Technol. 174:117-151 (2002).

[14] Duan X.R., Meier W., Weigand P., Lehmann B., "Phase-Resolved Laser Raman Scattering and Laser Doppler Velocimetry Applied to Periodic Instabilities in a Gas Turbine Model Combustor", Appl. Phys. B 80:389-396 (2005).

[15] Bergmann V., Meier W., Wolff D., Stricker W., "Application of Spontaneous Raman and Rayleigh Scattering and 2D LIF for the Characterization of a Turbulent $\mathrm{CH}_{4} / \mathrm{H}_{2} / \mathrm{N}_{2}$ Jet Diffusion Flame", Appl. Phys. B 66:489-502 (1998).

[16] Chen J.Y., UC Berkeley, California, USA, private communication. 
[17] Masri A.R., Kalt P.A.M., Barlow R.S., "The Compositional Structure of SwirlStabilised Turbulent Nonpremixed Flames”, Combust. Flame 137: 1-37 (2004).

[18] Meier W., Duan X.R., Weigand P., "Investigations of Swirl Flames in a Gas Turbine Model Combustor. Part II: Turbulence- Chemistry Interactions", Combust. Flame 144:225-236 (2006).

[19] Wehr L., Meier W., Kutne P., Hassa C., "Single-Pulse 1D Laser Raman Scattering Applied in a Gas Turbine Model Combustor at Elevated Pressure", Proc. Combust. Inst. 31: 3099-3107 (2007).

[20] Gregor M.A., Seffrin F., Fuest F., Geyer D., Dreizler A., "Multi-scalar measurements in a premixed swirl burner using 1D Raman/Rayleigh scattering”, Proc. Combust. Inst. 33: 1732-1746 (2009).

[21] Sweeney M.S., Hochgreb S., Dunn M.J., Barlow R.S., "The structure of turbulent stratified and premixed methane/air flames II: Swirling flows", Combust. Flame 159: 2912-2929 (2012).

[22] Kojima J., Fischer D.G., "Multiscalar analyses of high-pressure swirl-stabilized combustion via single-shot dual-SBG Raman spectroscopy", Combust. Sci. Technol. 185: 1735-1761 (2013).

[23] Stopper U., Meier W., Sadanandan R., Stöhr M., Aigner M., Bulat G., "Experimental study of industrial gas turbine flames including quantification of pressure influence on flow field, fuel/air premixing and flame shape“, Combust. Flame 160:2103-2118 (2013).

[24] Barlow R.S., J.H. Frank J.H., "Effects of Turbulence on Species Mass Fractions in Methane/Air Jet Flames", Proc. Combust. Inst. 27: 1087-1095 (1998). 